PROCEEDINGS OF THE

AMERICAN MATHEMATICAL SOCIETY

Volume 125, Number 8, August 1997, Pages 2391-2394

S 0002-9939(97)04108-7

\title{
ON REDUCIBILITY OF SEMIGROUPS OF COMPACT QUASINILPOTENT OPERATORS
}

\author{
ROMAN DRNOVŠEK
}

(Communicated by Palle E. T. Jorgensen)

\begin{abstract}
The following generalization of Lomonosov's invariant subspace theorem is proved. Let $\mathcal{S}$ be a multiplicative semigroup of compact operators on a Banach space such that $\hat{r}\left(S_{1}, \ldots, S_{n}\right)=0$ for every finite subset $\left\{S_{1}, \ldots, S_{n}\right\}$ of $\mathcal{S}$, where $\hat{r}$ denotes the Rota-Strang spectral radius. Then $\mathcal{S}$ is reducible.

This result implies that the following assertions are equivalent:

(A) For each infinite-dimensional complex Hilbert space $\mathcal{H}$, every semigroup of compact quasinilpotent operators on $\mathcal{H}$ is reducible.

(B) For every complex Hilbert space $\mathcal{H}$, for every semigroup of compact quasinilpotent operators on $\mathcal{H}$, and for every finite subset $\left\{S_{1}, \ldots, S_{n}\right\}$ of $\mathcal{S}$ it holds that $\hat{r}\left(S_{1}, \ldots, S_{n}\right)=0$.

The question whether the assertion (A) is true was considered by Nordgren, Radjavi and Rosenthal in 1984, and it seems to be still open.
\end{abstract}

Let $X$ be a (real or complex) Banach space and $\mathcal{B}(X)$ the Banach algebra of all bounded linear operators on $X$. Let $\left(S_{1}, \ldots, S_{n}\right)$ be an $n$-tuple of operators of $\mathcal{B}(X)$. The Rota-Strang spectral radius $\hat{r}\left(S_{1}, \ldots, S_{n}\right)$ is defined by

$$
\hat{r}\left(S_{1}, \ldots, S_{n}\right)=\lim _{m \rightarrow \infty} \max \left\{\left\|S_{k_{1}} S_{k_{2}} \cdots S_{k_{m}}\right\|^{1 / m}: 1 \leq k_{i} \leq n, 1 \leq i \leq m\right\} .
$$

In [7] it is shown that this limit always exists. Similarly, we introduce the local spectral radius of $n$-tuple $\left(S_{1}, \ldots, S_{n}\right)$ at a vector $x \in X$ by the formula

$$
\hat{r}\left(S_{1}, \cdots, S_{n} ; x\right)=\limsup _{m \rightarrow \infty} \max \left\{\left\|S_{k_{1}} S_{k_{2}} \cdots S_{k_{m}} x\right\|^{1 / m}: 1 \leq k_{i} \leq n, 1 \leq i \leq m\right\} .
$$

Let $\mathcal{C}$ be a collection of operators of $\mathcal{B}(X)$. We say that $\mathcal{C}$ is reducible if there exists a closed subspace of $X$, other than $\{0\}$ and $X$, which is invariant under every member of $\mathcal{C}$. The collection $\mathcal{C}$ is irreducible if it is not reducible. If there exists even a maximal subspace chain (i.e., a maximal totally ordered set of closed subspaces) whose elements are invariant under every member of $\mathcal{C}$, then $\mathcal{C}$ is said to be simultaneously triangularizable. Let us define the number $\hat{r}(\mathcal{C}) \in[0, \infty]$ by

$$
\hat{r}(\mathcal{C})=\sup \left\{\hat{r}\left(S_{1}, \ldots, S_{n}\right): n \in \mathbb{N}, S_{1}, \ldots, S_{n} \in \mathcal{C}\right\} .
$$

Similarly, we set, for any $x \in X$,

$$
\hat{r}(\mathcal{C} ; x)=\sup \left\{\hat{r}\left(S_{1}, \ldots, S_{n} ; x\right): n \in \mathbb{N}, S_{1}, \ldots, S_{n} \in \mathcal{C}\right\} .
$$

Received by the editors March 1, 1996.

1991 Mathematics Subject Classification. Primary 47A15, 47D03.

Key words and phrases. Invariant subspaces, semigroups, reducibility, simultaneous triangularizability.

This work was supported in part by the Research Ministry of Slovenia. 
The proof of our first result is based on the famous Lomonosov-Hilden technique; see e.g. [4].

Theorem 1. Let $X$ be a Banach space of dimension greater than one, and let $\mathcal{S}$ be a multiplicative semigroup in $\mathcal{B}(X)$ containing a compact operator $K$. If $\hat{r}\left(\mathcal{S} K ; x_{0}\right)=0$ and $K x_{0} \neq 0$ for some vector $x_{0} \in X$, then $\mathcal{S}$ is reducible.

Proof. There is no loss of generality in assuming that $\|K\|=1$, since we can assume that $\mathcal{S}=\mathbb{R}^{+} \mathcal{S}$. Replacing $x_{0}$ by $\lambda x_{0}$ for an appropriate scalar $\lambda>0$, we can also assume that $\left\|x_{0}\right\|>1$ and $\left\|K x_{0}\right\|>1$. Let $U=\left\{x \in X:\left\|x-x_{0}\right\| \leq 1\right\}$ be the closed unit ball centered at $x_{0}$. Obviously, 0 is not in $U$ nor in the closure $\overline{K(U)}$ of $K(U)$.

Let $\mathcal{A}$ be the linear manifold in $\mathcal{B}(X)$ generated by $\mathcal{S}$. Since $\mathcal{S}$ is a semigroup, $\mathcal{A}$ is equal to the subalgebra of $\mathcal{B}(X)$ generated by $\mathcal{S}$. For each fixed $y \in X$ the set

$$
L_{y}=\{A y: A \in \mathcal{A}\}
$$

is a linear manifold in $X$ which is invariant under every member of $\mathcal{A}$. If $L_{y}=\{0\}$ for some $y \neq 0$, then the one-dimensional subspace generated by $y$ is invariant under every member of $\mathcal{S}$. So, we may assume that $L_{y} \neq\{0\}$ for all $y \neq 0$. We shall show that there exists $y \neq 0$ such that $L_{y}$ is not dense in $X$. This will prove the theorem. Assume on the contrary that $L_{y}$ is dense in $X$ for all $y \neq 0$. Then for each $y \neq 0$ there is an $A \in \mathcal{A}$ such that $A y \in \operatorname{int} U$, where $\operatorname{int} U$ denotes the interior of $U$. We therefore have

$$
\overline{K(U)} \subseteq X \backslash\{0\} \subseteq \bigcup_{A \in \mathcal{A}} A^{-1}(\operatorname{int} U)
$$

Since $\overline{K(U)}$ is a compact set in $X$, there exists a finite set $\left\{A_{1}, \ldots, A_{n}\right\} \subseteq \mathcal{A}$ such that

$$
\overline{K(U)} \subseteq \bigcup_{i=1}^{n} A_{i}^{-1}(\operatorname{int} U)
$$

Therefore, it follows from $K x_{0} \in K(U)$ that $K x_{0} \in A_{i_{1}}^{-1}(U)$ for some $i_{1} \in\{1, \ldots, n\}$, and hence $A_{i_{1}} K x_{0} \in U$. Then $K A_{i_{1}} K x_{0} \in K(U)$ implies that $K A_{i_{1}} K x_{0} \in A_{i_{2}}^{-1}(U)$ for some $i_{2}$, and so $A_{i_{2}} K A_{i_{1}} K x_{0} \in U$. Proceeding with this "ping-pong" of Hilden's, after $m$ steps we obtain an integer $i_{m} \in\{1,2, \ldots, n\}$ such that

$$
A_{i_{m}} K A_{i_{m-1}} K \cdots A_{i_{1}} K x_{0} \in U .
$$

Clearly there exist $S_{1}, S_{2}, \ldots, S_{N} \in \mathcal{S}$ and scalars $c_{j}^{(i)}, i=1, \ldots, n, j=1, \ldots, N$, such that

$$
A_{i}=\sum_{j=1}^{N} c_{j}^{(i)} S_{j}
$$

for all $i=1, \ldots, n$. Set $c=\max \left\{\left|c_{j}^{(i)}\right|: i=1, \ldots, n, j=1, \ldots, N\right\}$. Putting $B_{i}=A_{i} K$ for $i=1, \ldots, n$, we then have

$$
\left\|B_{i_{m}} B_{i_{m-1}} \cdots B_{i_{1}} x_{0}\right\| \leq c^{m} N^{m} \max \left\{\left\|\left(S_{k_{m}} K\right) \cdots\left(S_{k_{1}} K\right) x_{0}\right\|: 1 \leq k_{i} \leq N\right\} .
$$

Since $\hat{r}\left(S_{1} K, \ldots, S_{N} K ; x_{0}\right)=0$, we conclude that

$$
\lim _{m \rightarrow \infty}\left\|B_{i_{m}} B_{i_{m-1}} \cdots B_{i_{1}} x_{0}\right\|^{1 / m}=0
$$


which implies that

$$
\lim _{m \rightarrow \infty}\left\|B_{i_{m}} B_{i_{m-1}} \cdots B_{i_{1}} x_{0}\right\|=0
$$

and so $0 \in U$. This contradiction completes the proof of the theorem.

In [2] an irreducible semigroup of nilpotent operators of index two is constructed. Let us use that semigroup to show that the compactness hypothesis of Theorem 1 cannot be omitted.

Example 2. If $A$ is a $k \times k$ matrix, then let $N_{A}$ be the $2 k \times 2 k$ matrix

$$
\left[\begin{array}{ll}
A & -A \\
A & -A
\end{array}\right]
$$

and let $T_{A}$ be the direct sum of denumerably many copies of $N_{A}$. We regard $T_{A}$ as an operator on a separable Hilbert space $l^{2}$.

For each $i \in \mathbb{N}$ let $\mathcal{S}_{i}$ denote the linear space of all operators $T_{A}$ as $A$ ranges over all $2^{i-1} \times 2^{i-1}$ matrices, and let $\mathcal{S}$ be the union of all $\mathcal{S}_{i}$. It is shown in [2] that $\mathcal{S}$ is an irreducible semigroup of nilpotent operators of index two. In order to show that $\hat{r}(\mathcal{S})=0$, let $\left(S_{1}, \ldots, S_{n}\right)$ be an $n$-tuple of non-zero operators of $\mathcal{S}$. Let $i\left(S_{p}\right)$ $(1 \leq p \leq n)$ denote the uniquely determined number $i$ such that $S_{p}$ belongs to $\mathcal{S}_{i}$. We claim that

$$
S_{k_{1}} S_{k_{2}} \cdots S_{k_{m}}=0
$$

for all $m \geq 2^{n}$ and for all $1 \leq k_{i} \leq n$. Indeed, there exist $1 \leq p<q \leq m$ such that $k_{p}=k_{q}$ and $i\left(S_{k_{j}}\right)<i\left(S_{k_{p}}\right)$ for each $p<j<q$. Using the facts that $\mathcal{S}_{i} \mathcal{S}_{j} \subseteq \mathcal{S}_{i}$ and $\mathcal{S}_{j} \mathcal{S}_{i} \subseteq \mathcal{S}_{i}$ for all $i>j$ and that $\mathcal{S}_{i} \mathcal{S}_{i}=\{0\}$ for all $i$, we now conclude that $S_{k_{p}} \cdots S_{k_{q}}=0$, which proves the claim. It follows that $\hat{r}\left(S_{1}, \ldots, S_{n}\right)=0$, so that $\hat{r}(\mathcal{S})=0$.

A recent extension of Rota's theorem [3, Corollary 1] gives the following result that will be needed in the sequel.

Proposition 3. Let $\mathcal{H}$ be a complex Hilbert space, and let $\mathcal{C}$ be a collection of compact quasinilpotent operators on $\mathcal{H}$ which is simultaneously triangularizable. Then $\hat{r}(\mathcal{C})=0$.

Proof. Let $\left(S_{1}, \ldots, S_{n}\right)$ be an $n$-tuple of operators of $\mathcal{C}$, and let $\epsilon>0$. By [3, Corollary 1] there exists a positive invertible operator $T$ such that $\left\|T^{-1} S_{k} T\right\|<\epsilon$ for all $k=1,2, \ldots, n$. We then have

$$
\left\|S_{k_{1}} \cdots S_{k_{m}}\right\| \leq\|T\|\left\|T^{-1} S_{k_{1}} T\right\| \cdots\left\|T^{-1} S_{k_{m}} T\right\|\left\|T^{-1}\right\|<\|T\|\left\|T^{-1}\right\| \epsilon^{m}
$$

for every $m \in \mathbb{N}$ and every $k_{1}, \ldots, k_{m} \in\{1, \ldots, n\}$. It follows that $\hat{r}\left(S_{1}, \ldots, S_{n}\right) \leq$ $\epsilon$. Therefore, $\hat{r}\left(S_{1}, \ldots, S_{n}\right)=0$, and so $\hat{r}(\mathcal{C})=0$.

Let $\mathcal{H}$ denote an arbitrary complex Hilbert space, and let $\mathbf{S}(\mathcal{H})$ be the collection of all multiplicative semigroups of compact quasinilpotent operators on $\mathcal{H}$.

Theorem 4. The following assertions are equivalent:

(a) For each $\mathcal{H}$ of dimension greater than one, every semigroup $\mathcal{S} \in \mathbf{S}(\mathcal{H})$ is reducible.

(b) For each $\mathcal{H}$, every semigroup $\mathcal{S} \in \mathbf{S}(\mathcal{H})$ is simultaneously triangularizable.

(c) $\hat{r}(\mathcal{S})=0$ for every $\mathcal{H}$ and for every $\mathcal{S} \in \mathbf{S}(\mathcal{H})$.

(d) $\hat{r}\left(\mathcal{S} ; x_{0}\right)=0$ for every $\mathcal{H}$, for every $\mathcal{S} \in \mathbf{S}(\mathcal{H})$, and for every $x_{0} \in \mathcal{H}$. 
(e) For each $\mathcal{H}$ and for each $\mathcal{S} \in \mathbf{S}(\mathcal{H})$ there exists a non-zero vector $x_{0} \in \mathcal{H}$ such that $\hat{r}\left(\mathcal{S} ; x_{0}\right)=0$.

Proof. The idea of the proof that (a) implies (b) is well-known. Since the proof is (for example) a slight modification of the proof of Corollary 1 in [5], we will omit it. By Proposition 3, (b) implies (c). It is easy to show that (c) implies (d) and that (d) implies (e).

Assume that (e) holds. Given $\mathcal{H}$ of dimension greater than one and $\mathcal{S} \in \mathbf{S}(\mathcal{H})$, there exists a non-zero vector $x_{0} \in \mathcal{H}$ such that $\hat{r}\left(\mathcal{S} ; x_{0}\right)=0$. If $S x_{0}=0$ for all $S \in \mathcal{S}$, then the one-dimensional subspace generated by $x_{0}$ is invariant under every member of $\mathcal{S}$, so that $\mathcal{S}$ is reducible. Otherwise, there is a $K \in \mathcal{S}$ such that $K x_{0} \neq 0$. Since $\hat{r}\left(\mathcal{S} K ; x_{0}\right)=0, \mathcal{S}$ is reducible by Theorem 1 , and so (a) holds.

Note that the problem (a) of the last result was studied in [5], where it is shown that every semigroup of trace class quasinilpotent operators is reducible and consequently simultaneously triangularizable.

We conclude by mentioning that the assertion (c) of Theorem 4 is not true if the compactness hypothesis is dropped. Namely, Guinand [1] constructed two weighted shifts $T_{1}$ and $T_{2}$ on $l^{2}$ of norm 1 such that every word in $\left\{T_{1}, T_{2}\right\}$ is nilpotent of index three. In other words, the semigroup $\mathcal{S}$ generated by $\left\{T_{1}, T_{2}\right\}$ consists of nilpotent operators on $l^{2}$. It was shown in [6, example] that $\hat{r}\left(T_{1}, T_{2}\right)=1$, which implies that $\hat{r}(\mathcal{S})=1$.

\section{ACKNOWLEDGEMENTS}

The author is grateful to Professor M. Omladič and Professor P. Rosenthal for their useful comments on the first version of the paper.

\section{REFERENCES}

1. P. S. Guinand, On quasinilpotent semigroups of operators, Proc. Amer. Math. Soc. 86 (1982), 485-486. MR 84h:47042

2. D. Hadwin, E. Nordgren, M. Radjabalipour, H. Radjavi and P. Rosenthal, A nil algebra of bounded operators on Hilbert space with semisimple norm closure, Integral Equat. Oper. Th. 9 (1986), 739-743. MR 87k:47104

3. A. A. Jafarian, H. Radjavi, P. Rosenthal and A. R. Sourour, Simultaneous triangularizability, near commutativity and Rota's theorem, Trans. Amer. Math. Soc. 347 (1995), 2191-2199. MR 95i: 47033

4. A. J. Michaels, Hilden's simple proof of Lomonosov's invariant subspace theorem, Adv. in Math. 25 (1977), 56-58. MR 59:17893

5. E. Nordgren, H. Radjavi and P. Rosenthal, Triangularizing semigroups of compact operators, Indiana Univ. Math. J. 33 (1984), 271-275. MR 85b:47047

6. P. Rosenthal and A. Soltysiak, Formulas for the joint spectral radius of non-commuting Banach algebra elements, Proc. Amer. Math. Soc. 123 (1995), 2705-2708. MR 95k:47008

7. G.-C. Rota and W. G. Strang, A note on the joint spectral radius, Indag. Math. 22 (1960), 379-381. MR 26:5434

Institute of Mathematics, Physics and Mechanics, Jadranska 19, 1000 Ljubljana, Slovenia

E-mail address: roman.drnovsek@fmf.uni-lj.si 